\title{
La violencia desde la perspectiva infantil: una lectura comparativa de Cartucho de Nellie Campobello y Fiesta en la madriguera de Juan Pablo Villalobos
}

\author{
Brigitte Adriaensen \\ Radboud Universiteit Nijmegen
}

En la producción literaria reciente, es llamativa la recurrencia de novelas sobre la guerra y la violencia escritas desde la perspectiva de un niño. ${ }^{1}$ Sin entrar ahora siquiera en el terreno del cine, en el campo de la literatura latinoamericana nada más se pueden mencionar ejemplos como La casa de los conejos (2008), de la franco-argentina Laura Alcoba, Papi (2005), de la escritora dominicana Rita Indiana Hernández, o Fiesta en la madriguera, (2010) del mexicano Juan Pablo Villalobos.

La lectura de la novela de Villalobos, que versa sobre el narcotráfico mexicano, muestra que dicha perspectiva no es tampoco exclusiva de la literatura contemporánea, ya que Fiesta en la madriguera trae a la memoria otro relato, esta vez sobre la Revolución Mexicana, que es Cartucho de Nellie Campobello, publicado en 1931. En una entrevista con Maria Fresquet, el propio autor acabó por confirmar esta impresión de intertextualidad. Dejando de lado los otros intertextos mencionados por Villalobos, como Un mundo para Julius, de Bryce Echenique, o A Catcher in the Rye, de Salinger, voy a limitarme en lo que sigue a un análisis comparativo entre Cartucho y Fiesta en la madriguera. ${ }^{2}$

A primera vista, puede parecer extrańo comparar dos textos procedentes de contextos tan disímiles, tanto desde el punto de vista histórico como literario. La obra de Campobello, por una parte, se relaciona con la novela de la Revolución por su temática. Para algunos críticos (Parra 57), la autora escribió la crónica de lo que pasó en el estado de Chihuahua entre 1916 y 1920, aproximadamente, la época más violenta de la Revolución mexicana. Sin embargo, hay dos diferencias esenciales entre Cartucho y el género literario con el cual se asocia la obra: primero, Campobello no escribió una novela propiamente dicha, sino más bien una crónica, unas estampas, o un relato autoficcional según la perspectiva de los diferentes críticos. En segundo lugar, su postura difiere de aquella, ya que "la perspectiva adulta, masculina, teleológica y pesimista de esta narrativa se ve destronada de su monopolio literario por un contrapunto de vista infantil, femenino, anecdótico, y, [...] por la visión lúdica de una niña que percibe la Revolución a partir de las coordenadas de su tierna edad" (Vanden Berghe 69). 
Villalobos, por su parte, publica Fiesta en la madriguera durante el sexenio de Felipe Calderón, que fue la época en que la violencia relacionada con el narcotráfico recrudeció tristemente y se hizo una exposición cada vez más lúgubre, pública y mediatizada de los cuerpos mutilados. Al igual que otras novelas sobre el narcotráfio, el texto también representa los "crímenes expresivos" que define Jean Franco de modo siguiente en su libro Cruel Modernity:

Expressive crimes are those in which bodies illustrate the logic of the killers. In the case of the murdered women in Ciudad Juárez, it is clear that, as female workers in Mexican assembly plants, they disrupted what had been a sexual division of labor. The mutilated and beheaded male bodies, on the other hand, are revenge killings that speak of treachery and betrayal. Thus both the murder of women and the beheading of males intentionally publicize the persistence of archaic codes. (21)

Como ella apunta, la dimensión icónica de la crueldad fue especialmente prominente durante el periodo de La Violencia en Colombia, y más tarde estas prácticas "passed with the drug traffic into Mexico, where cruelty is at its most extreme and where the expressive use of the cadaver has become common practice, a form of macabre theater addressed not only to rivals but also to the public" (Franco 14-15). La violencia relacionada con el combate al narcotráfico es un tema esencial de la llamada 'narconovela', cuyo auge en México desde los años 90 es conocido, y también controvertido. No han faltado los críticos que se han opuesto al género, ora por lo que consideraban su baja calidad literaria (Lemus, "Balas de salva") ora por su carácter comercial (Palaversich "La narcoliteratura") y su falta de ética con respecto al dolor de las víctimas de la violencia (Palaversich “¿Cómo hablar del silencio?”). Es interesante observar en este contexto el esfuerzo tanto de Juan Pablo Villalobos ${ }^{3}$ como del prologuista de la traducción al inglés, Adam Thirlwell, por alejar la obra del denostado género de la narcoliteratura:

Narcoliteratura is druglords and guns and girls. It is a corrupt and lurid politics. And while it might at first look like this miniature novel by the Mexican novelist Juan Pablo Villalobos belongs to the second category of literary history - the pulp category of narcoliteratura, since its protagonists are a druglord and his psychopathic minions - it really belongs to the first: the history of experiments. And so then: this is it. This novel is narrated by a kid called Tochtli, the son of a druglord. And I suppose that the usual story, in this narco era, would be a story of drugs and police and gangs. But since this is a story narrated by a kid, it is therefore not at all a contribution to narcoliteratura. (Thirlwell 27-35)

En los análisis que siguen, sin embargo, quedará claro que el narcotráfico no es un detalle del decorado novelístico. Pero lo que interesa rescatar por ahora es que tanto Cartucho como Fiesta en la madriguera recurren a la perspectiva infantil para hablar de la violencia que predominaba en sus contextos respectivos. En las próximas páginas, quisiera comparar los dos textos brevemente introducidos aquí, centrándome en tres puntos básicamente: el 
lugar y el modo de enunciación infantil, la representación de la violencia extrema de la guerra, y el papel del juego en la narración.

Empecemos por Cartucho de Nellie Campobello. Como destacamos anteriormente, es un libro-no exactamente una novela-que consta de 33 estampas sobre la Revolución Mexicana. Estas estampas están escritas en su mayoría desde la perspectiva de una niña, que se supone que es la propia autora, a una edad temprana. El relato cuenta la guerra sangrienta y cruel que destroza el pueblo de su infancia, el Parral, situado en Chihuahua. No sólo se oponen villistas y carrancistas, la guerra acaba por oponer a los mismos aldeanos e incluso a los miembros de una misma familia. Aunque en las últimas dos décadas parece haber una especie de auge en los estudios sobre Campobello, el libro tuvo una acogida muy tibia a la hora de publicarse. Generalmente los críticos aducen los mismos motivos: para empezar, el hecho de que Nellie Campobello fuera una mujer no contribuyó al prestigio de la novela. Además, su procedencia geográfica excéntrica no favorecía una aceptación favorable por parte de los intelectuales defeños. Otro motivo sería que en Cartucho se retrata de manera positiva a los villistas, en un momento en que Pancho Villa se veía fuertemente vilipendiado por el establishment. Y por último, a través de la perspectiva infantil, se enfocaba la guerra desde una perspectiva lúdica, otorgándole además una dimensión estética que estaba en las antípodas del acercamiento habitual. Resulta llamativo pues que desde los años 90, Cartucho sí haya gozado de una gran acogida crítica, lo cual podría deberse a que la sensibilidad estética de Campobello sea más afín a nuestros parámetros estéticos que a los de sus contemporáneos.

Como indica Jorge Aguilar Mora en su prólogo al libro, la posición enunciativa del relato es peculiar. Si bien la niña parece tener unos diez años aproximadamente, la propia Campobello tenía entre 15 y 20 años en el momento de la guerra (1905-1920), según la fecha de nacimiento que se adopte (1900 o 1909). La mirada infantil es reconstruida así en dos sentidos: la autora escribe el relato con la edad de 31 ańos aproximadamente, y escribe como si hubiera vivido los acontecimientos de niña, mientras en realidad ya había alcanzado la edad de una adolescente. Además, el texto vacila entre la perspectiva infantil y la adulta. Aunque la primera es predominante, de vez en cuando se incluyen referencias a la visión "ingenua" de la niña, tomando así cierta distancia con respecto al enfoque infantil e intercalando la perspectiva de la autora adulta $(71,81,104)$. Varios críticos han insistido además en la importancia de la memoria colectiva en el texto, a través de la incorporación de leyendas sobre Pancho Villa y sus hombres (p. ej. Parra 1998). En este contexto conviene enfatizar que si bien hay una dimensión legendaria en el relato, ésta no cobra una envergadura anónima, sino que viene muy arraigada en la especificidad del lugar, de la región del Parral en Chihuahua. En cada una de las estampas se especifica el lugar de origen del combatiente y el lugar donde se desarrolla la acción. De este modo, la pertenencia a la colectividad regional es muy prominente en la narración de Campobello.

En el caso de Fiesta en la madriguera, de Juan Pablo Villalobos, el niño narrador no nos habla pues de la Revolución Mexicana, sino de la violencia inherente a los conflictos originados por el negocio del narcotráfico. La novela consta de tres partes, que recuerdan la estructura de una novela de formación: la primera se desarrolla en el palacio de Yolcaut, el rey, donde Tochtli, su hijo, pasa todos los días. En la segunda parte, Tochtli cuenta el viaje, junto con su padre y su maestro, a Liberia, para capturar un hipopótamo enano que 
debe lustrar su colección de animales. La última parte muestra la maduración del niño, que después de un distanciamiento de su padre vuelve a acercársele y es iniciado a la edad adulta. Totchli entonces nos cuenta su vida desde dentro del palacio de su papá, un narco importante. Igual que la pequeña Nellie, el niño se enfrenta diariamente a la violencia: a través de la televisión, donde ve regularmente cabezas cortadas y otros restos humanos representados, o en el mismo palacio, donde aparecen ciertos sujetos que son enemigos de la pandilla, y que son maltratados, a veces delante de los ojos del niño, asesinados, y después entregados a los tigres.

A diferencia de Cartucho, la novela de Villalobos tuvo una recepción excelente y fue traducida a múltiples lenguas, como el inglés, el francés, el portugués, el alemán, el holandés y el rumano. Los motivos del éxito de la novela podrían ser varios. En primer lugar, el narcotráfico vende bien en el extranjero: viene a inscribirse en la tendencia de lo que Sánchez Prado ha llamado la "exotización de la violencia latinoamericana” en el mercado europeo y estadounidense. Fenómeno que en los años 30 no se daba todavía, al contrario, describir la barbarie de la revolución era algo inaudito y para el establishment, inaceptable. ${ }^{4}$

Otro motivo del éxito comercial de Fiesta en la madriguera podría ser la comicidad de la perspectiva del niño Tochtli. De hecho, tiene tendencia a usar lo que el propio autor ha llamado una "perspectiva hiperlógica", que a menudo se convierte en absurda. Cuando al niño le duele la panza, por ejemplo, el médico dice que se debe a la ausencia de su madre, y que debería ir a ver un sicólogo. Su reacción es ilustrativa: "Supuestamente eso se llama estar enfermo del psicosomos, que quiere decir que la enfermedad es de la mente. Pero yo no estoy enfermo de la mente, a mí nunca me ha dolido el cerebro" (48). La intención, en este sentido, es más claramente cómica que en el caso de Campobello. Especialmente el hecho de que Cartucho sea una narración autoficticia ${ }^{5}$ contribuye también a que su dimensión trágica sea mucho más palpable que en el caso de Fiesta en la madriguera, que es una novela enteramente ficcional. ${ }^{6}$ Eso no impide, por otra parte, que también en Cartucho haya una dimensión claramente cómica: fue analizada por Max Parra como un mecanismo de defensa, tanto típico de la cultura popular mexicana, como de la psique infantil, que intenta negar la muerte mediante la burla y la risa carnavalesca (179). Si bien se puede aducir el mismo argumento para explicar el sentido del humor en Villalobos, el hecho de que las circunstancias autobiográficas de ambos autores fueran tan diferentes, hace que también la risa produzca un efecto muy diverso. ${ }^{7}$

Por último, hay que ańadir que la novela de Villalobos no comparte el regionalismo de Cartucho, e incluso puede leerse al margen del género de la narcoliteratura, desde el prisma del Bildungsroman, tal como se propone en el prólogo de Adam Thirlwell a la traducción inglesa. A la composición fragmentaria de Cartucho se opone la coherencia de la trayectoria vital de Tochtli en Fiesta en la madriguera. Pero si bien el viaje que hace Tochtli a Liberia podría insinuar que la novela de Villalobos rehúye todo tipo de regionalismo, esto no es sino apariencia. El centro de la narración en esta novela es incluso más reducido y asfixiante que el de Cartucho. En vez de encontrarnos en el pueblo de El Parral, Villalobos nos muestra el lugar todavía más claustrofóbico del palacio, rodeado de vallas y de cámaras de seguridad. Tochtli dice conocer sólo a 14 personas. Añade que la suya es la mejor pandilla en un terreno de nada menos que ocho kilómetros. Pero a diferencia 
de Cartucho, donde se indicaba a cada rato dónde se situaba el frente, en qué pueblo nos encontrábamos, de dónde eran oriundos cada uno de los soldados y de los fusilados, aquí los topónimos faltan por completo. Lo único que sabemos es que estamos en México. Todo es secreto, todo es anónimo. Sin embargo, la impresión del niño de hacer una "vida de pandilla” se queda implícitamente ironizada. De hecho, la novela parece insinuar así que si bien la guerra de los narcos es una cuestión de pandillas, también es una guerra a escala nacional, que se repite idénticamente cada ocho kilómetros. Al hacer referencia además a los "socios" del rey, que son principalmente el "gober" y el gringo, al mismo tiempo se indica el alcance nacional e incluso internacional del rey. En este sentido, la violencia de los narcos en esta novela no se puede pensar separadamente de la violencia ejercida por el estado mismo. El narco y el estado son dos entidades que no se pueden disociar, lo cual en el caso de Campobello era bien diferente, ya que ella habla claramente de los villistas como los enemigos de las tropas nacionales.

Sigamos un momento con Cartucho de Campobello. En ese relato, el lugar de enunciación resulta extremadamente híbrido, y doblemente marginal. Primero, porque toma partido por los villistas, lo cual era una posición inusitada en los treintas. De hecho, en Cartucho se justifica la violencia de Pancho Villa como una violencia legítima, de defensa, contra la violencia ejercida por el mismo estado. La violencia revolucionaria se describe pues como único recurso ante el poder, en términos casi benjaminianos. Segundo, porque se percibe la guerra desde la perspectiva de la niña y de las mujeres (la madre es otro personaje central en todo el relato), actores marginales en el desarrollo tanto como en la descripción de la guerra. Como indica Max Parra (168), Campobello nos ofrece la voz de los subalternos sobre la guerra: ¿cuál fue la visión, la situación, la experiencia de estos nińos y estas mujeres, a menudo viudas o solteras, cómo vivieron la guerra desde los márgenes de la lucha?

En Villalobos, en cambio, hay cierto escepticismo ante la creación de una perspectiva subalterna, al contrario de lo que pasa en Cartucho. En Fiesta en la madriguera no vemos la guerra desde los márgenes del conflicto, sino desde el mismo palacio de los narcos. La violencia revolucionaria no se da en Villalobos como una alternativa para cuestionar el poder hegemónico. Se entiende que en el narco-estado mexicano ya no hay lugar inmaculado. No hay voz desde afuera. El niño, en este caso, tampoco es un subalterno en el sentido estricto: si bien en la primera parte parece ocupar esta posición, al igual que por ejemplo el músico en la novela Trabajos del reino de Yuri Herrera, poco a poco su pertenencia a la pandilla se va consolidando. Pero a diferencia del músico de Herrera, Tochtli no acaba distanciándose del régimen narco, sino al contrario, se integra plenamente.

En la pandilla, por cierto, las mujeres ocupan el mismo lugar marginal que en la guerra de los treintas. Los personajes femeninos que surgen en Fiesta en la madriguera son bien cocineras, bien sirvientas, bien prostitutas. La ausencia de la mamá de Tochtli es simbólica para la ausencia de figuras maternas en este mundo. El machismo lleva la voz cantante, pero, a diferencia de lo que pasa en Cartucho, no se ve contrarrestado por la perspectiva femenina. La novela de Villalobos no pretende tampoco dar voz al subalterno, es demasiado cínica para ello. No hay escape a la visión machista de los sicarios, se repiten los esquemas al cien por cien. A diferencia de ciertos narcocorridos, que supuestamente traducen la "voz del pueblo", que recurre al narcotráfico por culpa del estado corrupto que 
no crea empleo para los pobres, es interesante ver que en muchas narconovelas, tanto en esta de Villalobos como en Trabajos del reino de Yuri Herrera, por ejemplo, no hay lugar para la subalternidad dentro de la esfera del narco. El narco es omnipotente y manda al estado, no presenta ningún poder revolucionario.

Otro punto interesante para la comparación es la representación de los cuerpos destrozados por la guerra en ambos relatos. En primer lugar, el espectáculo de la violencia ya está muy presente en Cartucho. Se describen muchos cadáveres, que se asocian con un olor putrefacto, con la mugre (que es el título de una de las estampas), con la sangre, los orines: "estaba boca abajo, el cabello revuelto, sucio, las manos anchas, morenas. Las uñas negras, tenía en la espalda doblado un sarape gris, se veía ahogado de mugre” (75). Los cadáveres a primera vista se asocian pues con lo abyecto, definido por Kristeva de la manera siguiente:

[...] Tal como en un teatro verdadero, [...] sin máscara, el desecho como cadáver me indica lo que descarto permanentemente para vivir. Estos humores, esta suciedad, esta mierda con lo que soporta la vida apenas y con pena de la muerte. Ante el cadáver estoy en los límites de mi condición como ser vivo. De estos límites se libera mi cuerpo como vivo. (11)

Kristeva precisamente argumenta que en las sociedades modernas, el cadáver-y lo abyecto en general — queda fuera de la vista porque desafía el orden y la autoridad, nos vuelve a plantear nuestra vulnerabilidad: devuelve el cuerpo a su materialidad pura y produce asco y repulsión. De manera análoga a lo que ocurre en Fiesta en la madriguera, la dimensión abyecta de los cadáveres sangrientos, de los cuerpos destrozados, se ve resaltada por la perspectiva infantil. Kristine Vanden Berghe ya observó al respecto que en vez de asco y repulsión, la voz de la narradora expresa "impasibilidad frente a las mutilaciones sufridas por los cuerpos, que, las más de las veces, describe de un modo que delata un ojo expermentado" (53). Es el caso de la estampa titulada "Las tripas del general Sobarzo":

Como a las tres de la tarde, por la calle de San Francisco, estábamos en la piedra grande. Al bajar el callejón de la Pila de don Cirilo Reyes, vimos venir unos soldados con una bandeja en alto; pasaban junto a nosotras, iban platicando y riéndose. “¿igan, qué es eso tan bonito que llevan?” Desde arriba del callejón podíamos ver que dentro del lavamanos había algo color de rosa bastante bonito. Ellos se sonrieron, bajaron la bandeja y nos mostraron aquello. "Son tripas", dijo el más joven clavando sus ojos sobre nosotras a ver si nos asustábamos; al oír, son tripas, nos pusimos junto de ellos y las vimos; estaban enrolladitas como si no tuvieran punta. “¡Tripitas, qué bonitas!, ¿y de quién son?" dijimos con la curiosidad en el filo de los ojos. "De mi general Sobarzodijo el mismo soldado-, las llevamos a enterrar al camposanto". Se alejaron con el mismo pie todos, sin decir nada más. (85)

Para explicar la amoralidad y la impasibilidad ante el cadáver abyecto Vanden Berghe leyó Cartucho desde el ensayo Homo Ludens del holandés Johan Huizinga, demostrando así que la niña percibe la guerra en términos esencialmente lúdicos: representa a los soldados como 
si estuvieran "jugando" sobre sus caballos, haciendo "travesuras", riéndose con frecuencia. Además, según Huizinga: "El juego está fuera de la disyunción sensatez y necedad; pero fuera también del contraste verdad y falsedad, bondad y maldad. Aunque el jugar es una actividad espiritual, no es, por sí, una función moral, ni se dan en él virtud o pecado" (18-19). De esta manera, el juego y la mirada infantil se refuerzan en esa aproximación amoral y estética de la guerra.

En cuanto a la representación de la violencia en Villalobos, por lo general no vemos sino los resultados indirectos de la guerra: cabezas cortadas en la televisión, sangre que mancha el suelo del palacio, dos tigres que se dedican a comer cadáveres. A diferencia de Cartucho, el narrador de Fiesta en la madriguera no se ve sumergido en los estragos que hace la guerra entre la población. No hay escenas de lucha, apenas hay una escena donde se maltrata a un "enemigo", no hay viudas. Por lo general el relato transcurre en el mismo lugar, en la tranquilidad engañosa del palacio. De la violencia cruel omnipresente en Cartucho no vemos huellas aquí, aunque también a través del televisor la violencia hace su entrada a millones de hogares. Pero ya nos encontramos ante una violencia mediatizada. Por otra parte, igual que Nellie, Tochtli adopta un discurso distante, descriptivo sobre la violencia, donde lo abyecto carece de impacto y donde lo estético ocupa el primer plano:

Hoy hubo un cadáver enigmático en la tele: le cortaron la cabeza y ni siquiera se trataba de un rey. Tampoco parece que fuera obra de los franceses, que gustan tanto de cortar las cabezas. Los franceses ponen las cabezas en una cesta después de cortarlas. Lo miré en una película. En la guillotina colocan una cesta justo debajo de la cabeza del rey. Luego los franceses dejan caer la navaja y la cabeza cortada del rey cae en la cesta. Por eso me caen bien los franceses, que son tan delicados. [...] Los mexicanos no usamos cestas para las cabezas cortadas. Nosotros entregamos las cabezas cortadas en una caja de brandy añejo. [...] En la tele pasaron una foto de la cabeza y la verdad es que tenía un peinado muy feo. Llevaba el pelo largo y unas mechas pintadas de güero, patético. (42-43)

Obviamente, el lugar de enunciación de ambos niños no podría ser más distante: si la niña Nellie contemplaba la guerra como testigo, desde los márgenes, Tochtli la observa en la tele, como espectáculo, pero también la vive en su propio entorno, al formar parte de la misma pandilla (aunque sin ser, por ahora, un miembro activo). De alguna manera, es cómplice. La violencia ha permeado el microcosmos donde vive, hasta la médula, como se observa en el siguiente fragmento:

El juego es de preguntas y respuestas. Uno dice una cantidad de balas en una parte del cuerpo y el otro contesta: vivo, cadáver o pronóstico reservado. "Una bala en el corazón”. "Cadáver". "Treinta balazos en la uña del dedo chiquito del pie izquierdo". "Vivo". "Tres balazos en el páncreas". "Pronóstico reservado".

La misma falta de sensibilidad que se le reprochaba en su tiempo a la narradora de Campobello, quien describía la guerra sin compadecer a los muertos, se podría alegar en el caso de Tochtli. Pero a diferencia de lo que ocurre en Campobello, donde la niña describe 
el juego de la guerra desde fuera, sin comprender sus consecuencias realmente, en Fiesta en la madriguera Tochtli no sólo observa sino participa en el juego. Como explica Felipe Oliver, el juego no es inocente, pues es una manera de aprender cuáles son los límites del poder:

Lo que para Yolcaut puede resumirse a un simple juego, para Tochtli es una verdadera curiosidad científica (cuando se le acaban las partes acude a la enciclopedia) por conocer los límites del cuerpo, por establecer la frontera en donde la violencia transforma al "vivo" en "cadáver". El conocimiento puesto al servicio de la crueldad; el saber es poder y el poder se reconoce como tal cuando ejerce sistemáticamente la violencia en contra del cuerpo del otro. Por consiguiente, conocer los límites del cuerpo es conocer los límites del poder. El juego se convierte entonces en un aprendizaje, un proceso de formación que prepara a Tochtli para cargar en el futuro la estafeta que conduce el padre en el presente. (25)

A diferencia de lo que ocurre en Cartucho, donde la narradora contempla los estragos de la guerra desde su inocencia y su amoralidad infantil, en el caso de Villalobos la guerra es un juego desde dentro del poder, donde Tochtli toma el papel de partícipe, más que de testigo. Además, en Villalobos tampoco hay cabida para la violencia revolucionaria, como queda patente al final del libro. Tochtli en varias ocasiones indica que tiene un gran respeto por los franceses, por usar el método de la decapitación de manera tan profesional. Está claro que la novela de alguna manera ironiza esta admiración que tiene el niño por la civilización francesa, que opone a menudo a la manera más bruta, menos sofisticada en que los mexicanos cortan las cabezas. Ellos usan machetes, por lo cual necesitan darle varios golpes a la víctima, y además no guardan la cabeza en una cesta, sino en una caja de brandy añejo. Sin embargo, la burla de este ideal de la civilización llega a su colmo al final de la historia. Me refiero al momento en que Tochtli recibe dos cajas de cartón en las cuales se encuentran las cabezas disecadas de sus dos hipopótamos enanos de Liberia, llamados por él Luis XVI y María Antonieta, que habían muerto poco después de ser capturados. La imagen final parece alegórica:

La verdad, los disecadores hicieron un trabajo muy pulcro. Las cabezas cortadas tienen el hocico abierto para mostrar la lengua y sus cuatro colmillos. Además brillan, porque los disecadores las barnizaron con pintura transparente. Sus ojos están hechos de canicas blancas con la pupila color café. Y tienen las orejas minúsculas intactas. (103-104)

La insistencia en la higiene, en el trabajo pulcro, en la entereza de las cabezas, sin sangre, sin lastimaduras del discurso de Tochtli, difiere sustancialmente de la perspectiva en Cartucho, donde los cadáveres eran seres queridos, que la niña adora, extraña, toca sin miedo. Pero esta oposición entre los cuerpos sangrientos, mugrosos, abyectos de la revolución en Cartucho y las cabezas disecadas de los hipopótamos en Fiesta en la madriguera además es síntoma de otra diferencia esencial. Si en Cartucho se defiende a los villistas de manera apasionada, asumiendo la crueldad y la barbarie de la guerra como un hecho necesario para 
luchar contra el estado, en Fiesta en la madriguera la violencia revolucionaria se ha quedado sin savia. Aunque la forma en que los franceses decapitaban a los reyes es ejemplar para Tochtli, por ser "civilizada", por representar el ideal de la modernidad, de la civilización, de la violencia higiénica no manchada por la barbarie azteca y mexicana, está claro que esta violencia revolucionaria no tiene ya ningún potencial en la novela. Al colgar las cabezas perfectamente disecadas de los dos hipopótamos enanos de Liberia, llamados Luis XVI y María Antonieta de Austria, se renuncia al ideal de la revolución. Estas cabezas conservadas como piezas de un museo, indican la vacuidad del discurso revolucionario en la guerra de y contra los narcos.

\section{Notas}

${ }^{1}$ El presente artículo se inserta en el marco del proyecto "The Politics of Irony in Contemporary Latin American Fiction on Violence", subvencionado por la Comisión Holandesa para la Investigación Científica (NWO), 2011-2016.

2 En mi artículo "El exotismo de la violencia ironizado: Fiesta en la madriguera de Juan Pablo Villalobos" he desarrollado un análisis más general de la novela, donde no integro la comparación con la obra de Campobello.

${ }^{3}$ En una entrevista con el periódico holandés Het Parool, con ocasión de la traducción neerlandesa de la novela, Villalobos declaró lo siguiente: "Fiesta en la madriguera es una historia sobre crecer y perder la inocencia infantil. Es más que nada un Bildungsroman, sobre cómo un niño en una situación extrema construye estrategias de protección para hacer la realidad más llevadera. Y sobre cómo se ve forzado a aprender a seguir los pasos de su padre. El narcotráfico no es sino un decorado". (Bertina 2011: s.p.; mi traducción, BA)

4 Vanden Berghe (2013) menciona varios motivos para explicar la recepción problemática de Cartucho: el hecho de ser mujer y ofrecer un retrato de la crueldad inherente a la Revolución, su admiración abierta hacia Villa, vilipendiado por el establishment, su procedencia geográfica excéntrica y su distancia de los medios intelectuales (comunistas) del momento, la difícil adscripción del texto a un género literario determinado (19-20, 166-171).

5 Seguimos aquí la definición que da Manuel Alberca del término, según la cual la autoficción se da cuando el narrador, el protagonista y el autor se identifican como un mismo individuo al interior del texto. La autoficción, así, se sitúa a caballo entre el género de la autobiografía y de la ficción generando una fuerte ilusión referencial a la vez que proponiendo un pacto ambiguo (autobiográfico y novelesco a la vez) con el lector (Alberca 295).

6 Betina Keizman (2007) afirma así que se trata de una "crónica familiar" o de "un relato autobiográfico desplazado" (36), y Kristine Vanden Berghe lo denomina como una "forma temprana de autoficción” (37). Si bien se puede cuestionar el carácter puramente testimonial de la obra, por el juego ingenioso con la ambigüedad de la narración, es inevitable que su dimensión autobiográfica es mayor que la de Villalobos, que vive en el extranjero y no experimentó la violencia en México de forma comparable a Campobello, ni pone en escena ni personajes ni escenarios reales de la guerra.

${ }^{7}$ Es llamativo que entre los motivos de la recepción problemática de Cartucho no se suela mencionar su tono lúdico. Es decir: el público mexicano no parecía haberse perturbado demasiado ante él, ya que predominan otros reproches. Cuando he leído fragmentos de Fiestas en la madriguera ante un público europeo y otro mexicano, en cambio, la diferencia más llamativa era que el europeo compartía el sentido de humor, mientras que el mexicano se indignaba ante él, y lo encontraba inapropiado y superficial. Sin duda, la diferente posición de enunciación de ambos autores_y su ethos, para hablar con Maingueneau—no es ajena a esa observación.

\section{Obras citadas}

Adriaensen, Brigitte. "El exotismo de la violencia ironizado: Fiesta en la madriguera de Juan Pablo Villalobos". Narrativas del crimen en América Latina. Eds. Brigitte Adriaensen \& Valeria Grinberg Pla. Berlín: LitVerlag, 2012. 155-167. Impreso.

Aguilar Mora, Jorge. "Prólogo y cronología". Cartucho. Relatos de la lucha en el norte de México. México: Era, 2012. 9-44. Impreso. 
Alberca, Manuel. El pacto ambiguo: de la novela autobiográfica a la autoficción. Madrid: Biblioteca Nueva, 2007. Impreso.

Alcoba, Laura. La casa de los conejos. Trad. Leopoldo Brizuela. Barcelona: Edhasa, 2008.

Bertina, Daniël. "Drugsbaron heeft een zoon. Interview met Juan Pablo Villalobos". Het Parool 27-VIII2011. Impreso.

Campobello, Nellie. Cartucho. Relatos de la lucha en el norte de México. México: Era, 2012.

Franco, Jean. Cruel Modernity. Durham: Duke UP, 2013. Impreso.

Fresquet, Maria. "Entrevista (mínima). Juan Pablo Villalobos". Quimera 324 (2010): 8-9. Impreso.

Herrera, Yuri Trabajos del reino. Cáceres: Editorial Periférica, 2008. Impreso.

Huizinga, Johan. Homo ludens. Madrid: Alianza, 2007. Impreso.

Indiana, Rita. Papi. San Juan: Ediciones Vértigo, 2005. Impreso.

Keizman, Betina. "Entre el testimonio y la autobiografía, Cartucho y la construcción de una memoria poética/política”. Cuadernos del CILLHA 9/9 (2007):35-40. Impreso.

Kristeva, Julia. Pouvoir de l'horreur. Essai sur l'abjection. Paris: Editions du Seuil, 1980.

Lemus, Rafael. "Balas de salva". Letras libres. Sep. 2005. Web. 15 junio 2015. Impreso.

Oliver Fuentes Kraffczyk, Felipe. Apuntes para una poética de la narcoliteratura. Guanajuato: Universidad de Guanajuato, 2013. Impreso.

Palaversich, Diana. "La narcoliteratura del margen al centro". Revista de Literatura Mexicana Contemporánea 16.43 (2009): 7-18. Impreso. - "¿Cómo hablar del silencio? Contrabando y Un vaquero cruza la frontera en silencio, dos casos ejemplares del acercamiento ético en la literatura mexicana sobre el narco". Ciberletras: journal of literary criticism and culture 29 (2012). N. pag. Web.

Parra, Max. "Memoria y guerra en Cartucho de Nellie Campobello". Revista de Crítica Literaria Latinoamericana 47 (1998): 167-186. Impreso.

Sánchez Prado, Ignacio. "Amores Perros: violencia exótica y miedo neoliberal". Revista de la Casa de las Américas 240 (2005): 139-153. Impreso.

Thirlwell, Adam. "Introduction". Down the Rabbit Hole. High Wycombe: And Other Stories, 2011. Versión Kindle.

Vanden Berghe, Kristine. Homo ludens en la Revolución. Una lectura de Nellie Campobello. Frankfurt/México: Vervuert/Bonilla Artigas Editores, 2013. Impreso.

Villalobos, Juan Pablo. Fiesta en la madriguera. Barcelona: Anagrama, 2010. Impreso. . Down the Rabbit Hole. Prologue by Adam Thirlwall. High Wycombe: And Other Stories, 2011. Versión Kindle. 